\title{
Emotional Distress
}

National Cancer Institute

\section{Source}

National Cancer Institute. Emotional Distress. NCI Thesaurus. Code C117162.

Disturbed mental state characterized by moodiness, irritability, agitation, anxiety, and/or depression resulting from physical or mental stress. 